Pacific

Journal of

Mathematics

\title{
AFFINE QUANTUM SCHUR ALGEBRAS \\ AND AFFINE HECKE ALGEBRAS
}

QIANG FU 


\title{
AFFINE QUANTUM SCHUR ALGEBRAS AND AFFINE HECKE ALGEBRAS
}

\author{
QIANG FU
}

\begin{abstract}
Let $F$ be the Schur functor from the category of finite-dimensional $\mathscr{H}_{\Delta}(r)_{\mathbb{C}^{-}}$ modules to that of finite-dimensional $\mathscr{S}_{\Delta}(n, r)_{\mathbb{C}}$-modules, where $\mathscr{H}_{\Delta}(r)_{\mathbb{C}}$ is the extended affine Hecke algebra of type $A$ over $\mathbb{C}$ and $\mathscr{S}_{\Delta}(n, r)_{\mathbb{C}}$ is the affine quantum Schur algebras over $\mathbb{C}$. The Drinfeld polynomials associated with $\mathrm{F}(V)$, where $V$ is an irreducible $\mathscr{H}_{\Delta}(r)_{\mathbb{C}}$-module, have been previously determined when $n>r$. Here we generalize these results to the case $n \leqslant r$. As an application, we recover the classification of finite-dimensional irreducible $\mathscr{S}_{\triangle}(\boldsymbol{n}, \boldsymbol{r})_{\mathbb{C}}$-modules proved by Deng, Du and Fu using a different method. As another application, we generalize a result of Green to the affine case.
\end{abstract}

\section{Introduction}

Finite-dimensional irreducible modules for quantum affine algebras were classified by Chari and Pressley $[1991 ; 1994 ; 1995 ; 1997]$ in terms of Drinfeld polynomials. Finite-dimensional irreducible modules for $\mathscr{H}_{\Delta}(r)_{\mathbb{C}}$ were classified in [Zelevinsky 1980; Rogawski 1985], where $\mathscr{H}_{\Delta}(r)_{\mathbb{C}}$ is the extended affine Hecke algebra of type $A$ over the complex field $\mathbb{C}$ with a non-root of unity. The category of finite-dimensional $\mathscr{H}_{\Delta}(r)_{\mathbb{C}}$-modules and the category of finite-dimensional $\mathrm{U}_{\mathbb{C}}\left(\widehat{\mathfrak{s}}_{n}\right)$-modules which are of level $r$ are related by a functor $\mathscr{F}$ defined in [Chari and Pressley 1996, §4.2]. Here $\mathrm{U}_{\mathbb{C}}\left(\mathfrak{s}_{n}\right)$ is quantum affine $\mathfrak{s l}_{n}$ over $\mathbb{C}$. Chari and Pressley [loc. cit.] proved that $\mathscr{F}_{F}$ is an equivalence of categories if $n>r$. Furthermore the Drinfeld polynomials associated with $\mathscr{F}(V)$ were determined in [loc. cit., §7.6] in the case of $n>r$, where $V$ is an irreducible $\mathscr{H}_{\Delta}(r) \mathbb{C}$-module.

Let $\mathrm{U}_{\mathbb{C}}\left(\widehat{\mathfrak{g l}}_{n}\right)$ be quantum affine $\mathfrak{g l}_{n}$ over $\mathbb{C}$. In [Frenkel and Mukhin 2002], finitedimensional irreducible polynomial representations of $U_{\mathbb{C}}\left(\widehat{\mathfrak{g}}_{n}\right)$ were classified. It was proved in [Deng, Du and Fu 2012, Theorem 3.8.1] that the natural algebra homomorphism $\zeta_{r}$ from $\mathrm{U}_{\mathbb{C}}\left(\widehat{\mathfrak{g l}}_{n}\right)$ to the affine quantum Schur algebra $\mathscr{S}_{\Delta}(n, r)_{\mathbb{C}}$ is

Supported by the National Natural Science Foundation of China, the Program NCET, Fok Ying Tung Education Foundation and the Fundamental Research Funds for the Central Universities.

MSC2010: 17B37, 20C08, 20G43.

Keywords: affine quantum Schur algebras, affine Hecke algebras, Schur functor. 
surjective. Every $\mathscr{Y}_{\Delta}(n, r)_{\mathbb{C}}$-module can be regarded as a $\mathrm{U}_{\mathbb{C}}\left(\widehat{\mathfrak{g l}}_{n}\right)$-module via $\zeta_{r}$. Let $\mathrm{F}$ be the Schur functor from the category of finite-dimensional $\mathscr{H}_{\Delta}(r)_{\mathbb{C}}$-modules

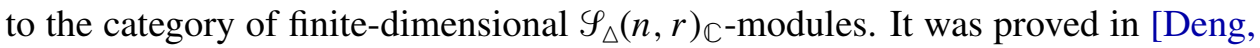
$\mathrm{Du}$ and $\mathrm{Fu}$ 2012, Theorem 4.1.3 and Proposition 4.2.1] that $\mathrm{F}$ is an equivalence of categories in the case of $n \geqslant r$ and that $\left.\mathrm{F}(V)\right|_{\mathrm{U}_{\mathbb{C}}\left(\widehat{\mathfrak{s}_{n}}\right)}$ is isomorphic to $\mathscr{F}(V)$ for any $\mathscr{H}_{\Delta}(r)_{\mathbb{C}}$-module $V$. Furthermore, using [Chari and Pressley 1996, §7.6], the Drinfeld polynomials associated with $\mathrm{F}(V)$ were determined in [Deng, Du and $\mathrm{Fu} 2012$, Theorem 4.4.2] in the case of $n>r$, where $V$ is an irreducible $\mathscr{H}_{\Delta}(r)_{\mathbb{C}}$-module. We will generalize these results to the case of $n \leqslant r$ in Theorem 4.9. Using this result, we will prove in Corollary 4.10 the classification theorem of finite-dimensional

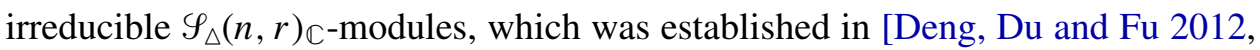
Theorem 4.6.8]. Finally, we will relate the parametrization of irreducible $\mathscr{T}_{\Delta}(N, r)_{\mathbb{C}^{-}}$ modules, via the functor $\mathrm{G}$ defined in (4.10.1), to the parametrization of irreducible

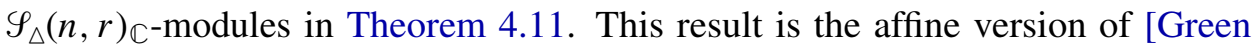
2007, (6.5f)].

\section{Quantum affine $\mathfrak{g l}_{n}$}

Let $v \in \mathbb{C}^{*}$ be a complex number which is not a root of unity, where $\mathbb{C}^{*}=\mathbb{C} \backslash\{0\}$. Let $\left(c_{i, j}\right)$ be the Cartan matrix of affine type $A_{n-1}$. We recall the Drinfeld's new realization of quantum affine $\mathfrak{g l}_{n}$ as follows.

Definition 2.1. The quantum loop algebra $\mathrm{U}_{\mathbb{C}}\left(\widehat{\mathfrak{g l}}_{n}\right)$ (or quantum affine $\mathfrak{g l}_{n}$ ) is the $\mathbb{C}$-algebra generated by $\mathrm{x}_{i, s}^{ \pm}(1 \leqslant i<n, s \in \mathbb{Z}), \mathrm{k}_{i}^{ \pm 1}$, and $\mathrm{g}_{i, t}(1 \leqslant i \leqslant n, t \in \mathbb{Z} \backslash\{0\})$ with the following relations:

(QLA1) $\mathrm{k}_{i} \mathrm{k}_{i}^{-1}=1=\mathrm{k}_{i}^{-1} \mathrm{k}_{i},\left[\mathrm{k}_{i}, \mathrm{k}_{j}\right]=0$,

(QLA2) $\mathrm{k}_{i} \mathrm{x}_{j, s}^{ \pm}=v^{ \pm\left(\delta_{i, j}-\delta_{i, j+1}\right)} \mathrm{x}_{j, s}^{ \pm} \mathrm{k}_{i},\left[\mathrm{k}_{i}, \mathrm{~g}_{j, s}\right]=0$,

(QLA3) $\left[\mathrm{g}_{i, s}, \mathrm{x}_{j, t}^{ \pm}\right]= \begin{cases}0 & \text { if } i \neq j, j+1, \\ \pm v^{-j s}([s] / s) \mathrm{x}_{j, s+t}^{ \pm} & \text {if } i=j, \\ \mp v^{-j s}([s] / s) \mathrm{x}_{j, s+t}^{ \pm} & \text {if } i=j+1,\end{cases}$

(QLA4) $\left[\mathrm{g}_{i, s}, \mathrm{~g}_{j, t}\right]=0$,

(QLA5) $\left[\mathrm{x}_{i, s}^{+}, \mathrm{x}_{j, t}^{-}\right]=\delta_{i, j}\left(\phi_{i, s+t}^{+}-\phi_{i, s+t}^{-}\right) /\left(v-v^{-1}\right)$,

(QLA6) $\mathrm{x}_{i, s}^{ \pm} \mathrm{x}_{j, t}^{ \pm}=\mathrm{x}_{j, t}^{ \pm} \mathrm{x}_{i, s}^{ \pm}$for $|i-j|>1$, and $\left[\mathrm{x}_{i, s+1}^{ \pm}, \mathrm{x}_{j, t}^{ \pm}\right]_{v^{ \pm c_{i j}}}=-\left[\mathrm{x}_{j, t+1}^{ \pm}, \mathrm{x}_{i, s}^{ \pm}\right]_{v^{ \pm c_{i j}}}$, (QLA7) $\left[\mathrm{x}_{i, s}^{ \pm},\left[\mathrm{x}_{j, t}^{ \pm}, \mathrm{x}_{i, p}^{ \pm}\right]_{v}\right]_{v}=-\left[\mathrm{x}_{i, p}^{ \pm},\left[\mathrm{x}_{j, t}^{ \pm}, \mathrm{x}_{i, s}^{ \pm}\right]_{v}\right]_{v}$ for $|i-j|=1$,

where $[x, y]_{a}=x y-a y x,[s]=\left(v^{s}-v^{-s}\right) /\left(v-v^{-1}\right)$, and the $\phi_{i, s}^{ \pm}$are defined via generating functions in the indeterminate $u$ by

$$
\Phi_{i}^{ \pm}(u):=\widetilde{\mathrm{k}}_{i}^{ \pm 1} \exp \left( \pm\left(v-v^{-1}\right) \sum_{m \geqslant 1} \mathrm{~h}_{i, \pm m} u^{ \pm m}\right)=\sum_{s \geqslant 0} \phi_{i, \pm s}^{ \pm} u^{ \pm s}
$$


with $\widetilde{\mathrm{k}}_{i}=\mathrm{k}_{i} / \mathrm{k}_{i+1}\left(\mathrm{k}_{n+1}=\mathrm{k}_{1}\right)$ and $\mathrm{h}_{i, \pm m}=v^{ \pm(i-1) m} \mathrm{~g}_{i, \pm m}-v^{ \pm(i+1) m} \mathrm{~g}_{i+1, \pm m}$ $(1 \leqslant i<n)$.

The algebra $\mathrm{U}_{\mathbb{C}}\left(\widehat{\mathfrak{g l}}_{n}\right)$ has another presentation which we now describe. Let $\mathfrak{D}_{\triangle, \mathbb{C}}(n)$ be the double Ringel-Hall algebra of the cyclic quiver $\triangle(n)$. By [Deng, $\mathrm{Du}$ and $\mathrm{Fu}$ 2012, Theorem 2.3.1], the algebra $\mathfrak{D}_{\triangle \mathbb{C}}(n)$ has the following presentation.

Lemma 2.2. The double Ringel-Hall algebra $\mathfrak{D}_{\triangle, \mathbb{C}}(n)$ of the cyclic quiver $\triangle(n)$ is the $\mathbb{C}$-algebra generated by $E_{i}, F_{i}, K_{i}, K_{i}^{-1}, \mathrm{z}_{s}^{+}, \mathrm{z}_{s}^{-}$, for $1 \leqslant i \leqslant n, s \in \mathbb{Z}^{+}$, and relations:

(QGL1) $K_{i} K_{j}=K_{j} K_{i}, K_{i} K_{i}^{-1}=1$,

(QGL2) $K_{i} E_{j}=v^{\delta_{i, j}-\delta_{i, j+1}} E_{j} K_{i}, K_{i} F_{j}=v^{-\delta_{i, j}+\delta_{i, j+1}} F_{j} K_{i}$,

(QGL3) $E_{i} F_{j}-F_{j} E_{i}=\delta_{i, j}\left(\widetilde{K}_{i}-\widetilde{K}_{i}^{-1}\right) /\left(v-v^{-1}\right)$, where $\widetilde{K}_{i}=K_{i} K_{i+1}^{-1}$,

(QGL4) $\sum_{a+b=1-c_{i, j}}(-1)^{a}\left[\begin{array}{c}1-c_{i, j} \\ a\end{array}\right] E_{i}^{a} E_{j} E_{i}^{b}=0$ for $i \neq j$,

(QGL5) $\sum_{a+b=1-c_{i, j}}(-1)^{a}\left[\begin{array}{c}1-c_{i, j} \\ a\end{array}\right] F_{i}^{a} F_{j} F_{i}^{b}=0$ for $i \neq j$,

(QGL6) $\mathrm{z}_{s}^{+} \mathrm{z}_{t}^{+}=\mathrm{z}_{t}^{+} \mathrm{z}_{s}^{+}, \mathrm{z}_{s}^{-} \mathrm{z}_{t}^{-}=\mathrm{z}_{t}^{-} \mathrm{z}_{s}^{-}, \mathrm{z}_{s}^{+} \mathrm{z}_{t}^{-}=\mathrm{z}_{t}^{-} \mathrm{z}_{s}^{+}$,

(QGL7) $K_{i} \mathrm{z}_{s}^{+}=\mathrm{z}_{s}^{+} K_{i}, K_{i} \mathrm{z}_{s}^{-}=\mathrm{z}_{s}^{-} K_{i}$,

(QGL8) $E_{i} \mathrm{z}_{s}^{+}=\mathrm{z}_{s}^{+} E_{i}, E_{i} \mathrm{z}_{s}^{-}=\mathrm{z}_{s}^{-} E_{i}, F_{i} \mathrm{z}_{s}^{-}=\mathrm{z}_{s}^{-} F_{i}$, and $\mathrm{z}_{s}^{+} F_{i}=F_{i} \mathrm{z}_{s}^{+}$,

where $1 \leqslant i, j \leqslant n, s, t \in \mathbb{Z}^{+}$, and

$$
\left[\begin{array}{l}
c \\
a
\end{array}\right]=\prod_{s=1}^{a} \frac{v^{c-s+1}-v^{-c+s-1}}{v^{s}-v^{-s}} \quad \text { for } c \in \mathbb{Z} .
$$

It is a Hopf algebra with comultiplication $\Delta$, counit $\varepsilon$, and antipode $\sigma$ defined by

$$
\begin{gathered}
\Delta\left(E_{i}\right)=E_{i} \otimes \widetilde{K}_{i}+1 \otimes E_{i}, \quad \Delta\left(F_{i}\right)=F_{i} \otimes 1+\widetilde{K}_{i}^{-1} \otimes F_{i}, \\
\Delta\left(K_{i}^{ \pm 1}\right)=K_{i}^{ \pm 1} \otimes K_{i}^{ \pm 1}, \quad \Delta\left(\mathrm{z}_{s}^{ \pm}\right)=\mathrm{z}_{s}^{ \pm} \otimes 1+1 \otimes \mathrm{z}_{s}^{ \pm}, \\
\varepsilon\left(E_{i}\right)=\varepsilon\left(F_{i}\right)=0=\varepsilon\left(\mathrm{z}_{s}^{ \pm}\right), \quad \varepsilon\left(K_{i}\right)=1, \\
\sigma\left(E_{i}\right)=-E_{i} \widetilde{K}_{i}^{-1}, \quad \sigma\left(F_{i}\right)=-\widetilde{K}_{i} F_{i}, \quad \sigma\left(K_{i}^{ \pm 1}\right)=K_{i}^{\mp 1}, \quad \sigma\left(\mathrm{z}_{s}^{ \pm}\right)=-\mathrm{z}_{s}^{ \pm},
\end{gathered}
$$

where $1 \leqslant i \leqslant n$ and $s \in \mathbb{Z}^{+}$.

Let $\mathrm{U}_{\mathbb{C}}\left(\widehat{\mathfrak{s}}_{n}\right)$ be the subalgebra of $\mathfrak{D}_{\triangle, \mathbb{C}}(n)$ generated by $E_{i}, F_{i}, \widetilde{K}_{i}, \widetilde{K}_{i}^{-1}$ for $i \in[1, n]$. Beck [1994] proved that $\mathrm{U}_{\mathbb{C}}\left(\widehat{\mathfrak{s}}_{n}\right)$ is isomorphic to the subalgebra of $\mathrm{U}_{\mathbb{C}}\left(\widehat{\mathfrak{g l}}_{n}\right)$ generated by all $\mathrm{x}_{i, s}^{ \pm}, \widetilde{\mathrm{k}}_{i}^{ \pm 1}$, and $\mathrm{h}_{i, t}$. The following result extends Beck's isomorphism. 
Lemma 2.3 [Deng, Du and Fu 2012, Proposition 4.4.1]. There is a Hopf algebra isomorphism

$$
f: \mathfrak{D}_{\triangle, \mathbb{C}}(n) \rightarrow \mathrm{U}_{\mathbb{C}}\left(\widehat{\mathfrak{g l}}_{n}\right)
$$

such that

$$
\begin{aligned}
& K_{i}^{ \pm 1} \mapsto \mathrm{k}_{i}^{ \pm 1}, \quad E_{j} \mapsto \mathrm{x}_{j, 0}^{+}, \quad F_{j} \mapsto \mathrm{x}_{j, 0}^{-} \quad(1 \leqslant i \leqslant n, 1 \leqslant j<n),
\end{aligned}
$$

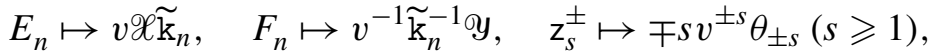

where

$$
\begin{aligned}
\theta_{ \pm s} & =\mp \frac{1}{[s]}\left(\mathrm{g}_{1, \pm s}+\cdots+\mathrm{g}_{n, \pm s}\right), \\
\mathscr{X} & =\left[\mathrm{x}_{n-1,0}^{-},\left[\mathrm{x}_{n-2,0}^{-}, \ldots,\left[\mathrm{x}_{2,0}^{-}, \mathrm{x}_{1,1}^{-}\right]_{v^{-1}} \cdots\right]_{v^{-1}}\right]_{v^{-1}}, \\
\mathscr{y} & =\left[\cdots\left[\left[\mathrm{x}_{1,-1}^{+}, \mathrm{x}_{2,0}^{+}\right]_{v}, \mathrm{x}_{3,0}^{+}\right]_{v}, \ldots, \mathrm{x}_{n-1,0}^{+}\right]_{v} .
\end{aligned}
$$

We now review the classification theorem of finite-dimensional irreducible polynomial $\mathrm{U}_{\mathbb{C}}\left(\widehat{\mathfrak{g}}_{n}\right)$-modules. We first need to introduce the elements $\mathscr{2}_{i, s} \in \mathrm{U}_{\mathbb{C}}\left(\widehat{\mathfrak{g l}}_{n}\right)$, which will be used to define pseudo-highest weight modules. For $1 \leqslant i \leqslant n$ and $s \in \mathbb{Z}$, define the elements $\mathscr{2}_{i, s} \in \mathrm{U}_{\mathbb{C}}\left(\widehat{\mathfrak{g}}_{n}\right)$ through the generating functions

$$
2_{i}^{ \pm}(u):=\exp \left(-\sum_{t \geqslant 1} \frac{1}{[t]} g_{i, \pm t}(v u)^{ \pm t}\right)=\sum_{s \geqslant 0} 2_{i, \pm s} u^{ \pm s} \in \mathrm{U}_{\mathbb{C}}\left(\widehat{g}_{n}\right)\left[\left[u, u^{-1}\right]\right] .
$$

For a representation $V$ of $\mathrm{U}_{\mathbb{C}}\left(\widehat{\mathfrak{g}}_{n}\right)$, a nonzero vector $w \in V$ is called a pseudo-highest weight vector if there exists some $Q_{i, s} \in \mathbb{C}$ such that

$$
\mathrm{x}_{j, s}^{+} w=0, \quad 2_{i, s} w=Q_{i, s} w, \quad \mathrm{k}_{i} w=v^{\lambda_{i}} w
$$

for all $1 \leqslant i \leqslant n$ and $1 \leqslant j \leqslant n-1$ and $s \in \mathbb{Z}$. The module $V$ is called a pseudo-highest weight module if $V=\mathrm{U}_{\mathbb{C}}\left(\widehat{\mathfrak{g}}_{n}\right) w$ for some pseudo-highest weight vector $w$. We also write the short form $2_{i}^{ \pm}(u) w=Q_{i}^{ \pm}(u) w$ for the relations $\mathscr{2}_{i, s} w=Q_{i, s} w(s \in \mathbb{Z})$, where

$$
Q_{i}^{ \pm}(u)=\sum_{s \geqslant 0} Q_{i, \pm s} u^{ \pm s}
$$

Let $V$ be a finite-dimensional polynomial representation of $U_{\mathbb{C}}\left(\widehat{g}_{n}\right)$ of type 1 . Then $V=\oplus_{\lambda \in \mathbb{N}^{n}} V_{\lambda}$, where

$$
V_{\lambda}=\left\{x \in V \mid \mathrm{k}_{j} x=v^{\lambda_{j}} x, 1 \leqslant j \leqslant n\right\},
$$

and, since all $\mathscr{2}_{i, s}$ commute with the $\mathrm{k}_{j}$, each $V_{\lambda}$ is a direct sum of generalized eigenspaces of the form

$$
V_{\lambda, \gamma}=\left\{x \in V_{\lambda} \mid\left(2_{i, s}-\gamma_{i, s}\right)^{p} x=0 \quad \text { for some } p(1 \leqslant i \leqslant n, s \in \mathbb{Z})\right\},
$$

where $\gamma=\left(\gamma_{i, s}\right)$ with $\gamma_{i, s} \in \mathbb{C}$. Let $\Gamma_{i}^{ \pm}(u)=\sum_{s \geqslant 0} \gamma_{i, \pm s} u^{ \pm s}$. 
A finite-dimensional $\mathrm{U}_{\mathbb{C}}\left(\widehat{\mathfrak{g l}}_{n}\right)$-module $V$ is called a polynomial representation if the restriction of $V$ to $\mathrm{U}_{\mathbb{C}}\left(\mathfrak{g l}_{n}\right)$ is a polynomial representation of type 1 and, for every weight $\lambda=\left(\lambda_{1}, \ldots, \lambda_{n}\right) \in \mathbb{N}^{n}$ of $V$, the formal power series $\Gamma_{i}^{ \pm}(u)$ associated to the eigenvalues $\left(\gamma_{i, s}\right)_{s \in \mathbb{Z}}$ defining the generalized eigenspaces $V_{\lambda, \gamma}$ as given in (2.3.2), are polynomials in $u^{ \pm}$of degree $\lambda_{i}$ so that the zeroes of the functions $\Gamma_{i}^{+}(u)$ and $\Gamma_{i}^{-}(u)$ are the same.

Following [Frenkel and Mukhin 2002], an $n$-tuple of polynomials

$$
\boldsymbol{Q}=\left(Q_{1}(u), \ldots, Q_{n}(u)\right)
$$

with constant terms 1 is called dominant if, for each $1 \leqslant i \leqslant n-1$, the ratio $Q_{i}\left(v^{i-1} u\right) / Q_{i+1}\left(v^{i+1} u\right)$ is a polynomial. Let $2(n)$ be the set of dominant $n$-tuples of polynomials.

For $g(u)=\prod_{1 \leqslant i \leqslant m}\left(1-a_{i} u\right) \in \mathbb{C}[u]$ with constant term 1 and $a_{i} \in \mathbb{C}^{*}$, define

$$
g^{ \pm}(u)=\prod_{1 \leqslant i \leqslant m}\left(1-a_{i}^{ \pm 1} u^{ \pm 1}\right) .
$$

For $\boldsymbol{Q}=\left(Q_{1}(u), \ldots, Q_{n}(u)\right) \in \mathcal{2}(n)$, define $Q_{i, s} \in \mathbb{C}$, for $1 \leqslant i \leqslant n$ and $s \in \mathbb{Z}$, by the formula

$$
Q_{i}^{ \pm}(u)=\sum_{s \geqslant 0} Q_{i, \pm s} u^{ \pm s}
$$

where $Q_{i}^{ \pm}(u)$ is defined using (2.3.3). Let $I(Q)$ be the left ideal of $\mathrm{U}_{\mathbb{C}}\left(\widehat{\mathfrak{g}}_{n}\right)$ generated by $\mathrm{x}_{j, s}^{+}, \mathscr{2}_{i, s}-Q_{i, s}$, and $\mathrm{k}_{i}-v^{\lambda_{i}}$, for $1 \leqslant j \leqslant n-1,1 \leqslant i \leqslant n$, and $s \in \mathbb{Z}$, where $\lambda_{i}=\operatorname{deg} Q_{i}(u)$, and define

$$
M(\boldsymbol{Q})=\mathrm{U}_{\mathbb{C}}\left(\widehat{\mathfrak{g l}}_{n}\right) / I(\boldsymbol{Q}) .
$$

Then $M(\boldsymbol{Q})$ has a unique irreducible quotient, denoted by $L(\boldsymbol{Q})$. The polynomials $Q_{i}(u)$ are called Drinfeld polynomials associated with $L(\boldsymbol{Q})$.

Theorem 2.4 [Frenkel and Mukhin 2002]. The $\mathrm{U}_{\mathbb{C}}\left(\widehat{\mathfrak{g l}}_{n}\right)$-modules $L(\boldsymbol{Q})$ with $\boldsymbol{Q} \in$ $2(n)$ are all nonisomorphic finite-dimensional irreducible polynomial representations of $\mathrm{U}_{\mathbb{C}}\left(\widehat{\mathfrak{g}}_{n}\right)$.

If $\boldsymbol{Q}, \boldsymbol{Q}^{\prime} \in 2(n)$ satisfies $Q_{j}\left(v^{j-1} u\right) / Q_{j+1}\left(v^{j+1} u\right)=Q_{j}^{\prime}\left(v^{j-1} u\right) / Q_{j+1}^{\prime}\left(v^{j+1} u\right)$ and $\operatorname{deg} Q_{j}(u)-\operatorname{deg} Q_{j+1}(u)=\operatorname{deg} Q_{j}^{\prime}(u)-\operatorname{deg} Q_{j+1}^{\prime}(u)$ for $1 \leqslant j \leqslant n-1$, then $\left.\left.L(\boldsymbol{Q})\right|_{\mathrm{U}_{\mathbb{C}}\left(\widehat{\left.\mathfrak{s}_{n}\right)}\right.} \cong L\left(\boldsymbol{Q}^{\prime}\right)\right|_{\mathrm{U}_{\mathbb{C}}\left(\widehat{\mathfrak{s}} \widehat{\mathfrak{s}}_{n}\right)}$, by [Deng, Du and Fu 2012, Lemma 4.7.1, Corollary 4.7.2]. Thus we can denote $\left.L(\boldsymbol{Q})\right|_{\mathrm{U}_{\mathbb{C}}\left(\widehat{\left.\mathfrak{s} \hat{\mathfrak{I}}_{n}\right)}\right.}$ by $\bar{L}(\boldsymbol{P})$, where $\boldsymbol{P}=\left(P_{1}(u), \ldots, P_{n-1}(u)\right)$ with $P_{j}(u)=Q_{j}\left(v^{j-1} u\right) / Q_{j+1}\left(v^{j+1} u\right)$.

Let $\mathscr{P}(n)$ be the set of $(n-1)$-tuples of polynomials with constant term 1 . The following result is due to Chari and Pressley [1991; 1994; 1995].

Theorem 2.5. The modules $\bar{L}(\boldsymbol{P})$ with $\boldsymbol{P} \in \mathscr{P}(n)$ are all nonisomorphic finitedimensional irreducible $\mathrm{U}_{\mathbb{C}}\left(\widehat{\mathfrak{s}}_{n}\right)$-modules of type 1 . 


\section{Affine quantum Schur algebras}

In this section we collect some facts about extended affine Hecke algebras and affine quantum Schur algebras, which will be used in Section 4. The extended affine Hecke algebra $\mathscr{H}_{\Delta}(r)_{\mathbb{C}}$ is defined to be the algebra generated by

$$
T_{i}, \quad X_{j}^{ \pm 1} \quad(1 \leqslant i \leqslant r-1,1 \leqslant j \leqslant r),
$$

and relations

$$
\begin{gathered}
\left(T_{i}+1\right)\left(T_{i}-v^{2}\right)=0, \\
T_{i} T_{i+1} T_{i}=T_{i+1} T_{i} T_{i+1}, \quad T_{i} T_{j}=T_{j} T_{i}(|i-j|>1), \\
X_{i} X_{i}^{-1}=1=X_{i}^{-1} X_{i}, \quad X_{i} X_{j}=X_{j} X_{i} \\
T_{i} X_{i} T_{i}=v^{2} X_{i+1}, \quad X_{j} T_{i}=T_{i} X_{j}(j \neq i, i+1) .
\end{gathered}
$$

Let $\mathfrak{S}_{r}$ be the symmetric group with generators $s_{i}:=(i, i+1)$ for $1 \leqslant i \leqslant r-1$. Let $I(n, r)=\left\{\left(i_{1}, \ldots, i_{r}\right) \in \mathbb{Z}^{r} \mid 1 \leqslant i_{k} \leqslant n, \forall k\right\}$. The symmetric group $\mathfrak{S}_{r}$ acts on the set $I(n, r)$ by place permutation:

$$
\boldsymbol{i} w=\left(i_{w(1)}, \ldots, i_{w(r)}\right), \quad \text { for } \boldsymbol{i} \in I(n, r) \text { and } w \in \mathfrak{S}_{r} .
$$

Let $\Omega_{\mathbb{C}}$ be a vector space over $\mathbb{C}$ with basis $\left\{\omega_{i} \mid i \in \mathbb{Z}\right\}$. For $\boldsymbol{i}=\left(i_{1}, \ldots, i_{r}\right) \in \mathbb{Z}^{r}$, write

$$
\omega_{i}=\omega_{i_{1}} \otimes \omega_{i_{2}} \otimes \cdots \otimes \omega_{i_{r}}=\omega_{i_{1}} \omega_{i_{2}} \cdots \omega_{i_{r}} \in \Omega_{\mathbb{C}}^{\otimes r}
$$

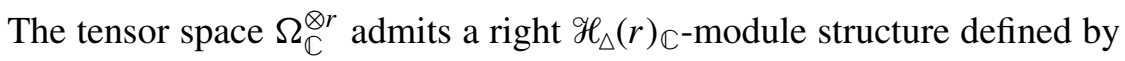

$$
\left\{\begin{array}{l}
\omega_{\boldsymbol{i}} \cdot X_{t}^{-1}=\omega_{i_{1}} \cdots \omega_{i_{t-1}} \omega_{i_{t}+n} \omega_{i_{t+1}} \cdots \omega_{i_{r}} \\
\omega_{\boldsymbol{i}} \cdot T_{k}=\left\{\begin{array}{ll}
v^{2} \omega_{i} & \text { if } i_{k}=i_{k+1}, \\
v \omega_{i s_{k}} & \text { if } i_{k}<i_{k+1}, \\
v \omega_{i s_{k}}+\left(v^{2}-1\right) \omega_{i} & \text { if } i_{k+1}<i_{k},
\end{array} \text { for all } \boldsymbol{i} \in I(n, r),\right.
\end{array}\right.
$$

where $1 \leqslant k \leqslant r-1$ and $1 \leqslant t \leqslant r$.

The algebra

$$
\mathscr{S}_{\Delta}(n, r)_{\mathbb{C}}:=\operatorname{End}_{\mathscr{H}_{\Delta}(r)_{\mathbb{C}}}\left(\mathscr{T}_{\Delta}(n, r)\right)
$$

is called an affine $q$-Schur algebra, where $\mathscr{T}_{\Delta}(n, r)=\Omega_{\mathbb{C}}^{\otimes r}$. Let $\Omega_{n, \mathbb{C}}$ be the subspace of $\Omega_{\mathbb{C}}$ spanned by $\omega_{i}$ with $1 \leqslant i \leqslant n$ and $\mathcal{H}_{(r}(r)_{\mathbb{C}}$ be the subalgebra of $\mathscr{H}_{\Delta}(r)_{\mathbb{C}}$ generated by $T_{k}$ for $1 \leqslant k \leqslant r-1$. Then the algebra $\mathscr{S}(n, r)_{\mathbb{C}}:=\operatorname{End}_{\mathscr{H}(r)_{\mathbb{C}}}(\mathscr{T}(n, r))$ is called a $q$-Schur algebra, where $\mathscr{T}(n, r)=\Omega_{n, \mathbb{C}}^{\otimes r}$.

The algebras $\mathrm{U}_{\mathbb{C}}\left(\widehat{\mathfrak{g}}_{n}\right)$ and $\mathscr{S}_{\Delta}(n, r)_{\mathbb{C}}$ are related by an algebra homomorphism $\zeta_{r}$, which we now describe. For $i \in \mathbb{Z}$, let $\bar{l}$ denotes the corresponding integer modulo $n$. 
The complex vector space $\Omega_{\mathbb{C}}$ is a natural $\mathfrak{D}_{\triangle \mathbb{C}}(n)$-module with the action

$$
\begin{gathered}
E_{i} \cdot \omega_{s}=\delta_{\overline{i+1}, \bar{s}} \omega_{s-1}, \quad F_{i} \cdot \omega_{s}=\delta_{\bar{l}, \bar{s}} \omega_{s+1}, \quad K_{i}^{ \pm 1} \cdot \omega_{s}=v^{ \pm \delta_{\bar{l}, \bar{s}}} \omega_{s}, \\
\mathrm{z}_{t}^{+} \cdot \omega_{s}=\omega_{s-t n}, \quad \mathrm{z}_{t}^{-} \cdot \omega_{s}=\omega_{s+t n} .
\end{gathered}
$$

The Hopf algebra structure induces a $\mathfrak{D}_{\triangle, \mathbb{C}}(n)$-module $\Omega_{\mathbb{C}}^{\otimes r}$. By [Deng, Du and Fu 2012, Proposition 3.5.5], the actions of $\mathfrak{D}_{\triangle, \mathbb{C}}(n)$ and $\mathscr{H}_{\Delta}(r)_{\mathbb{C}}$ on $\Omega_{\mathbb{C}}^{\otimes r}$ are commute. We will identify $\mathfrak{D}_{\triangle \mathbb{C}}(n)$ and $\mathrm{U}_{\mathbb{C}}\left(\widehat{\mathfrak{g l}}_{n}\right)$ via the algebra isomorphism $f$ defined in Lemma 2.3. Consequently, there is an algebra homomorphism

$$
\zeta_{r}: \mathrm{U}_{\mathbb{C}}\left(\widehat{\mathfrak{g}}_{n}\right)=\mathfrak{D}_{\triangle, \mathbb{C}}(n) \rightarrow \mathscr{S}_{\Delta}(n, r)_{\mathbb{C}}
$$

It is proved in [Deng, Du and Fu 2012, Theorem 3.8.1] that $\zeta_{r}$ is surjective. Let $\mathrm{U}_{\mathbb{C}}\left(\mathfrak{g l}_{n}\right)$ be the subalgebra of $\mathfrak{D}_{\triangle, \mathbb{C}}(n)$ generated by $E_{i}, F_{i}, K_{j}, K_{j}^{-1}$ for $1 \leqslant i \leqslant n-1$ and $1 \leqslant j \leqslant n$. The restriction of $\zeta_{r}$ to $\mathrm{U}_{\mathbb{C}}\left(\mathfrak{g l}_{n}\right)$ induces a surjective algebra homomorphism $\zeta_{r}: \mathrm{U}_{\mathbb{C}}\left(\mathfrak{g l}_{n}\right) \rightarrow \mathscr{Y}(n, r)_{\mathbb{C}}$ (see [Jimbo 1986]). Every $\mathscr{S}_{\Delta}(n, r)_{\mathbb{C}^{-}}$ module (resp., $\mathscr{S}(n, r)_{\mathbb{C}}$-module) will be inflated into a $\mathrm{U}_{\mathbb{C}}\left(\widehat{\mathfrak{g l}}_{n}\right)$-module (resp., $\mathrm{U}_{\mathbb{C}}\left(\mathfrak{g l}_{n}\right)$-module) via $\zeta_{r}$.

The following easy lemma relates $\Omega_{\mathbb{C}}^{\otimes r}$ with $\Omega_{n, \mathbb{C}}^{\otimes r}$.

Lemma 3.1 [Deng, Du and Fu 2012, Lemma 4.1.1]. There is a $\mathrm{U}_{\mathbb{C}}\left(\mathfrak{g l}_{n}\right)$ - $\mathscr{H}_{\Delta}(r)_{\mathbb{C}^{-}}$ bimodule isomorphism

$$
\Omega_{n, \mathbb{C}}^{\otimes r} \otimes_{\mathcal{H}(r)_{\mathbb{C}}} \mathscr{H}_{\Delta}(r)_{\mathbb{C}} \stackrel{\sim}{\longrightarrow} \Omega_{\mathbb{C}}^{\otimes r}, \quad x \otimes h \mapsto x h .
$$

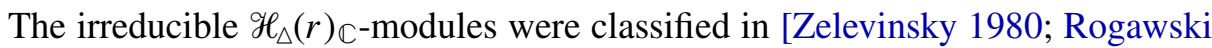
1985], which we now describe. For $\boldsymbol{a}=\left(a_{1}, \ldots, a_{r}\right) \in\left(\mathbb{C}^{*}\right)^{r}$, let $M_{\boldsymbol{a}}=\mathscr{H}_{\Delta}(r)_{\mathbb{C}} / J_{\boldsymbol{a}}$, where $J_{\boldsymbol{a}}$ is the left ideal of $\mathscr{H}_{\Delta}(r)_{\mathbb{C}}$ generated by $X_{j}-a_{j}$ for $1 \leqslant j \leqslant r$.

A segment $\mathrm{s}$ with center $a \in \mathbb{C}^{*}$ is by definition an ordered sequence

$$
\mathrm{s}=\left(a v^{-k+1}, a v^{-k+3}, \ldots, a v^{k-1}\right) \in\left(\mathbb{C}^{*}\right)^{k} .
$$

Here $k$ is called the length of the segment, denoted by $|s|$. If $s=\left\{s_{1}, \ldots, s_{p}\right\}$ is an unordered collection of segments, define $\wp(s)$ to be the partition associated with the sequence $\left(\left|s_{1}\right|, \ldots,\left|s_{p}\right|\right)$. That is, $\wp(s)=\left(\left|s_{i_{1}}\right|, \ldots,\left|s_{i_{p}}\right|\right)$ with $\left|s_{i_{1}}\right| \geqslant \cdots \geqslant\left|s_{i_{p}}\right|$, where $\left|s_{i_{1}}\right|, \ldots,\left|s_{i_{p}}\right|$ is a permutation of $\left|s_{1}\right|, \ldots,\left|s_{p}\right|$. We also call $|s|:=|\wp(s)|$ the length of $s$.

Let $\mathscr{Y}_{r}$ be the set of unordered collections of segments $s$ with $|s|=r$. Then $\mathscr{S}_{r}=\bigcup_{\mu \in \Lambda^{+}(r)} \mathscr{S}_{r, \mu}$, where $\mathscr{S}_{r, \mu}=\left\{s \in \mathscr{S}_{r} \mid \wp(s)=\mu\right\}$ and $\Lambda^{+}(r)$ is the set of partitions of $r$.

If $w=s_{i_{1}} s_{i_{2}} \cdots s_{i_{m}}$ is reduced let $T_{w}=T_{i_{1}} T_{i_{2}} \cdots T_{i_{m}}$. For $p \geqslant 1$ let

$$
\Lambda(p, r)=\left\{\mu \in \mathbb{N}^{p} \mid \sum_{1 \leqslant i \leqslant p} \mu_{i}=r\right\}
$$


For $\mu \in \Lambda(p, r)$ let $\mathfrak{S}_{\mu}$ be the corresponding standard Young subgroup of the symmetric group $\mathfrak{S}_{r}$, and let $\mathscr{D}_{\mu}=\left\{d \in \mathfrak{S}_{r} \mid \ell(w d)=\ell(w)+\ell(d)\right.$ for $\left.w \in \mathfrak{S}_{\mu}\right\}$. For $\mu \in \Lambda(p, r)$ let

$$
\Phi_{\mu}=\mathscr{H}(r)_{\mathbb{C}} y_{\mu}
$$

where

$$
y_{\mu}=\sum_{w \in \mathfrak{S}_{\mu}}\left(-v^{2}\right)^{-\ell(w)} T_{w} \in \mathscr{H}(r)_{\mathbb{C}} .
$$

For $s=\left\{\mathrm{s}_{1}, \ldots, \mathrm{s}_{p}\right\} \in \mathscr{Y}_{r, \mu}$, let $\boldsymbol{a}(\boldsymbol{s})=\left(\mathrm{s}_{1}, \ldots, \mathrm{s}_{p}\right) \in\left(\mathbb{C}^{*}\right)^{r}$ be the $r$-tuple obtained by juxtaposing the segments in $s$. Let $\iota: \mathscr{H}(r)_{\mathbb{C}} \rightarrow M_{\boldsymbol{a}(s)}$ be the natural $\mathscr{H}(r)_{\mathbb{C}}$-module isomorphism defined by sending $h$ to $\bar{h}$. Let

$$
\bar{\Phi}_{\mu}=\iota\left(\mathscr{I}_{\mu}\right)=\mathscr{H}(r)_{\mathbb{C}} \bar{y}_{\mu}=\mathscr{H}_{\Delta}(r)_{\mathbb{C}} \bar{y}_{\mu} .
$$

Then,

$$
\mathscr{H}(r)_{\mathbb{C}} y_{\mu} \cong E_{\mu} \oplus\left(\bigoplus_{\substack{\nu \vdash r \\ \nu \triangleright \lambda}} m_{\nu, \mu} E_{\nu}\right),
$$

where $E_{v}$ is the left cell module defined by the Kazhdan-Lusztig's C-basis [1979] associated with the left cell containing $w_{0, v}$.

Let $V_{s}$ be the unique composition factor of the $\mathscr{H}_{\Delta}(r)_{\mathbb{C}}$-module $\mathscr{H}_{\Delta}(r)_{\mathbb{C}} \bar{y}_{\mu}$ such that the multiplicity of $E_{\mu}$ in $V_{s}$ as an $\mathscr{H}(r)_{\mathbb{C}}$-module is nonzero.

The following classification theorem is due to [Zelevinsky 1980; Rogawski 1985].

Theorem 3.2. The modules $V_{s}$ with $s \in \mathscr{Y}_{r}$ are all nonisomorphic finite-dimensional irreducible $\mathscr{H}_{\Delta}(r)_{\mathbb{C}}$-modules.

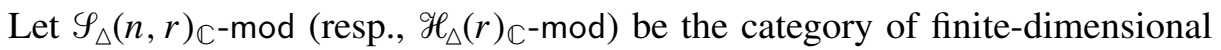

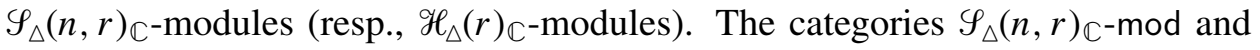

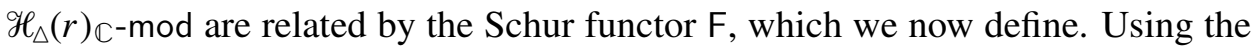
$\mathscr{S}_{\Delta}(n, r)_{\mathbb{C}}-\mathscr{H}_{\Delta}(r)_{\mathbb{C}}$-bimodule $\Omega_{\mathbb{C}}^{\otimes r}$, we define a functor

$$
\mathrm{F}=\mathrm{F}_{n, r}: \mathscr{H}_{\Delta}(r)_{\mathbb{C}}-\bmod \rightarrow \mathscr{S}_{\Delta}(n, r)_{\mathbb{C}}-\bmod , \quad V \mapsto \Omega_{\mathbb{C}}^{\otimes r} \otimes_{\mathscr{H}_{\Delta}(r)_{\mathbb{C}}} V .
$$

Let

$$
\mathscr{Y}_{r}^{(n)}=\left\{s=\left\{\mathrm{s}_{1}, \ldots, \mathrm{s}_{p}\right\} \in \mathscr{Y}_{r}, p \geqslant 1,\left|\mathrm{~s}_{i}\right| \leqslant n, \forall i\right\} .
$$

The following classification theorem is given in [Deng, Du and Fu 2012, Theorems 4.3.4 and 4.5.3].

Lemma 3.3. For $s \in \mathscr{Y}_{r}$ we have $\mathrm{F}\left(V_{s}\right) \neq 0$ if and only if $s \in \mathscr{S}_{r}^{(n)}$. Furthermore, the set

$$
\left\{\mathrm{F}\left(V_{\boldsymbol{s}}\right) \mid \boldsymbol{s} \in \mathscr{Y}_{r}^{(n)}\right\}
$$


is a complete set of nonisomorphic finite-dimensional irreducible $\mathscr{S}_{\Delta}(n, r) \mathbb{C}$-modules.

The following result, which will be used in Theorem 4.9, is taken from [Chari and Pressley 1996, §7.6; Deng, Du and Fu 2012, Theorem 4.4.2 and Lemma 4.6.5].

Lemma 3.4. Assume $n \geqslant r$. Let $\boldsymbol{s}=\left(a v^{-r+1}, a v^{-r+3}, \ldots, a v^{r-1}\right)$ be a single segment and $\mu=\wp(s)=(r)$. Then $V_{s}=\bar{\Phi}_{\mu}$ and $\mathrm{F}\left(V_{s}\right) \cong L(Q)$, where $\boldsymbol{Q}=$ $\left(Q_{1}(u), \ldots, Q_{n}(u)\right)$ with

$$
\begin{aligned}
Q_{n}(u) & =\left(1-a v^{-n+1} u\right)^{\delta_{n, r}}, \\
\frac{Q_{i}\left(u v^{i-1}\right)}{Q_{i+1}\left(u v^{i+1}\right)} & =(1-a u)^{\delta_{i, r}} \quad \text { for } 1 \leqslant i \leqslant n-1 .
\end{aligned}
$$

\section{Identification of irreducible $\mathscr{S}_{\triangle}(n, r)_{\mathbb{C}}$-modules}

In this section we will prove that $\mathrm{F}\left(\bar{\Phi}_{\wp(s)}\right)$ is isomorphic to the tensor product

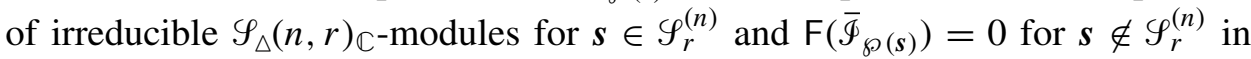
Proposition 4.6. Using this result, we will relate the parametrization of irreducible $\mathscr{H}_{\Delta}(r)_{\mathbb{C}}$-modules, via the functor $\mathrm{F}$ defined in (3.2.1), to the parametrization of finitedimensional irreducible polynomial representations of $U_{\mathbb{C}}\left(\widehat{\mathfrak{g l}}_{n}\right)$ in Theorem 4.9. As applications, we will classify finite-dimensional irreducible $\mathscr{Y}_{\Delta}(n, r)_{\mathbb{C}}$-modules in Corollary 4.10, and generalize [Green 2007, (6.5f)] to the affine case.

To compute $\mathrm{F}\left(\overline{\mathscr{\Phi}}_{\wp(s)}\right)$, we need Proposition 4.3 of [Rogawski 1985], which we now describe. For $1 \leqslant j \leqslant p$, let $\mathscr{H}_{\mu, j}$ be the subalgebra of $\mathscr{H}(r)_{\mathbb{C}}$ generated by $T_{i}$ with $s_{i} \in \mathfrak{S}_{\mu^{(j)}}$, where

$$
\mu^{(j)}=\left(1^{\mu_{[1, j-1]}}, \mu_{j}, 1^{r-\mu_{[1, j]}}\right),
$$

and $\mu_{[1, j]}=\mu_{1}+\mu_{2}+\cdots+\mu_{j}$. Since $\mathscr{H}_{\mu, j} \cong \mathscr{H}\left(\mu_{j}\right)_{\mathbb{C}}$ for $1 \leqslant j \leqslant p$ and $\Omega_{n, \mathbb{C}}^{\otimes \mu_{j}}$ is a right $\mathscr{H}\left(\mu_{j}\right)_{\mathbb{C}}$-module, $\Omega_{n, \mathbb{C}}^{\otimes \mu_{j}}$ can be also regarded as a right $\mathscr{H}_{\mu, j}$-module.

Recall the notation $\Phi_{\mu}$ defined in (3.1.2). For $\mu \in \Lambda(p, r)$ and $1 \leqslant j \leqslant p$ let

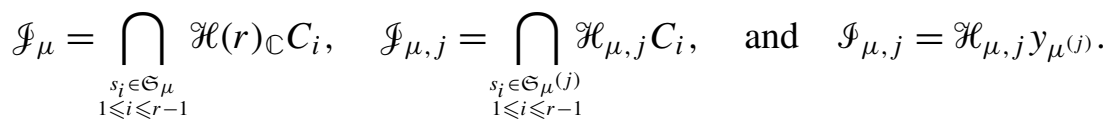

where $C_{i}=v^{-1} T_{i}-v$ and $y_{\mu^{(j)}}=\sum_{w \in \mathfrak{S}_{\mu}(j)}\left(-v^{2}\right)^{-\ell(w)} T_{w}$. By Proposition 4.3 of
[Rogawski 1985] we have:

Lemma 4.1. We have $\mathscr{I}_{\mu}=\mathscr{I}_{\mu}, \mathscr{I}_{\mu, j}=\mathscr{I}_{\mu, j}$ for $\mu \in \Lambda(p, r)$ and $1 \leqslant j \leqslant p$.

Lemma 4.2. Assume $I$ is a left ideal of $\mathscr{H}(r)_{\mathbb{C}}$. Then $\Omega_{n, \mathbb{C}}^{\otimes r} \otimes_{\mathscr{H}(r)_{\mathbb{C}}} I \cong \Omega_{n, \mathbb{C}}^{\otimes r} I$. 
Proof. Since $\mathscr{H}(r)_{\mathbb{C}}$ is semisimple, there exist a left ideal $J$ of $\mathscr{H}(r)_{\mathbb{C}}$ such that $\mathscr{H}(r)_{\mathbb{C}}=I \oplus J$. Then $\Omega_{n, \mathbb{C}}^{\otimes r} \cong \Omega_{n, \mathbb{C}}^{\otimes r} \otimes_{\mathcal{H}(r) \mathbb{C}} \mathscr{H}(r)_{\mathbb{C}} \cong \Omega_{n, \mathbb{C}}^{\otimes r} \otimes_{\mathcal{H}(r)_{\mathbb{C}}} I \oplus \Omega_{n, \mathbb{C}}^{\otimes r} \otimes_{\mathcal{H}(r)_{\mathbb{C}}} J$. Thus the natural linear map $f: \Omega_{n, \mathbb{C}}^{\otimes r} \otimes \mathscr{H}(r)_{\mathbb{C}} I \rightarrow \Omega_{n, \mathbb{C}}^{\otimes r}$ defined by sending $w \otimes h$ to wh is injective. Consequently, $\Omega_{n, \mathbb{C}}^{\otimes r} \otimes_{\mathscr{H}(r)_{\mathbb{C}}} I \cong \operatorname{Im}(f)=\Omega_{n, \mathbb{C}}^{\otimes r} I$.

By Lemmas 3.1, 4.1, and 4.2 we conclude that $\mathrm{F}\left(\overline{\mathscr{\Phi}}_{\mu}\right) \cong \Omega_{n, \mathbb{C}}^{\otimes r} \otimes \mathscr{H}(r) \mathbb{C} \overline{\mathscr{F}}_{\mu} \cong \Omega_{n, \mathbb{C}}^{\otimes r} \mathscr{F}_{\mu}$, where $\mu=\wp(s)$ for some $s \in \mathscr{S}_{r}$. We now compute $\Omega_{n, \mathbb{C}}^{\otimes r} \mathscr{F}_{\mu}$.

Lemma 4.3. For $\mu \in \Lambda(p, r)$, we have

$$
\Omega_{n, \mathbb{C}}^{\otimes r} \mathscr{F}_{\mu}=\Omega_{n, \mathbb{C}}^{\otimes \mu_{1}} \mathscr{F}_{\mu, 1} \otimes \cdots \otimes \Omega_{n, \mathbb{C}}^{\otimes \mu_{p}} \mathscr{F}_{\mu, p} .
$$

Proof. Since $\mathscr{F}_{\mu}=\bigcap_{1 \leqslant j \leqslant p} \mathscr{F}_{\mu^{(j)}}$ we have $\Omega_{n, \mathbb{C}}^{\otimes r} \mathscr{\mathscr { F }}_{\mu} \subseteq \bigcap_{1 \leqslant j \leqslant p}\left(\Omega_{n, \mathbb{C}}^{\otimes r} \mathscr{\mathscr { F }}_{\mu^{(j)}}\right)$. Furthermore by Lemma 4.1 we have $\mathscr{\mathscr { S }}_{\mu^{(j)}}=\mathscr{I}_{\mu^{(j)}}=\mathscr{X}_{\mu, j} \mathscr{I}_{\mu, j}=\mathscr{X}_{\mu, j} \mathscr{F}_{\mu, j}$ where $\mathscr{X}_{\mu, j}=\operatorname{span}\left\{T_{w} \mid w \in \mathscr{D}_{\mu^{(j)}}^{-1}\right\}$. This implies that

$$
\Omega_{n, \mathbb{C}}^{\otimes r} \mathscr{F}_{\mu^{(j)}}=\Omega_{n, \mathbb{C}}^{\otimes r} \mathscr{F}_{\mu, j}=\Omega_{n, \mathbb{C}}^{\mu_{1}} \otimes \cdots \otimes \Omega_{n, \mathbb{C}}^{\mu_{j-1}} \otimes \Omega_{n, \mathbb{C}}^{\otimes \mu_{j}} \mathscr{F}_{\mu_{j}} \otimes \Omega_{n, \mathbb{C}}^{\otimes \mu_{j+1}} \otimes \cdots \otimes \Omega_{n, \mathbb{C}}^{\otimes \mu_{p}}
$$

for $1 \leqslant j \leqslant p$. Thus,

$$
\begin{aligned}
& \Omega_{n, \mathbb{C}}^{\otimes r} \mathscr{F}_{\mu} \subseteq \bigcap_{1 \leqslant j \leqslant p}\left(\Omega_{n, \mathbb{C}}^{\mu_{1}} \otimes \cdots \otimes \Omega_{n, \mathbb{C}}^{\mu_{j-1}} \otimes \Omega_{n, \mathbb{C}}^{\otimes \mu_{j}} \mathscr{F}_{\mu_{j}} \otimes \Omega_{n, \mathbb{C}}^{\otimes \mu_{j+1}} \otimes \cdots \otimes \Omega_{n, \mathbb{C}}^{\otimes \mu_{p}}\right) \\
& =\Omega_{n, \mathbb{C}}^{\otimes \mu_{1}} \mathscr{F}_{\mu, 1} \otimes \cdots \otimes \Omega_{n, \mathbb{C}}^{\otimes \mu_{p}} \mathscr{F}_{\mu, p} .
\end{aligned}
$$

On the other hand, we assume $w_{1} h_{1} \otimes \cdots \otimes w_{p} h_{p} \in \Omega_{n, \mathbb{C}}^{\otimes \mu_{1}} \mathscr{F}_{\mu, 1} \otimes \cdots \otimes \Omega_{n, \mathbb{C}}^{\otimes \mu_{p}} \mathscr{F}_{\mu, p}$, where $w_{j} \in \Omega_{n, \mathbb{C}}^{\otimes \mu_{j}}$ and $h_{j} \in \mathscr{F}_{\mu, j}$. Since $h_{k} h_{l}=h_{l} h_{k}$ for any $k, l$ and $h_{j} \in \mathscr{F}_{\mu, j}$, we have $h_{1} h_{2} \cdots h_{p}=\left(h_{1} \cdots h_{j-1} h_{j+1} \cdots h_{p}\right) h_{j} \in \mathscr{H}(r)_{\mathbb{C}} \mathscr{F}_{\mu, j} \subseteq \mathscr{H}(r)_{\mathbb{C}} C_{i}$ for $1 \leqslant i \leqslant r-1,1 \leqslant j \leqslant p$ with $s_{i} \in \mathfrak{S}_{\mu^{(j)}}$. This implies that $h_{1} h_{2} \cdots h_{p} \in \mathscr{F}_{\mu}$. It follows that $w_{1} h_{1} \otimes \cdots \otimes w_{p} h_{p}=\left(w_{1} \otimes \cdots \otimes w_{p}\right) h_{1} \cdots h_{p} \in \Omega_{n, \mathbb{C}}^{\otimes r} \mathscr{F}_{\mu}$. The assertion follows.

For $\mu \in \Lambda(p, r)$ and $1 \leqslant j \leqslant p$, let $\widetilde{\mathscr{H}}_{\mu, j}$ be the subalgebra of $\mathscr{H}_{\Delta}(r)_{\mathbb{C}}$ generated by $T_{i}$ and $X_{\mu_{[1, j-1]}+1}, \ldots, X_{\mu_{[1, j]}}$ with $s_{i} \in \mathfrak{S}_{\mu^{(j)}}$. Since $\widetilde{\mathscr{H}}_{\mu, j} \cong \mathscr{H}_{\Delta}\left(\mu_{j}\right)_{\mathbb{C}}$ and $\Omega_{\mathbb{C}}^{\otimes \mu_{j}}$

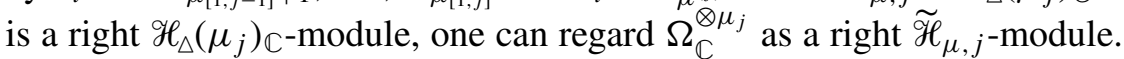

For $\boldsymbol{s}=\left\{\mathrm{s}_{1}, \ldots, \mathrm{s}_{p}\right\} \in \mathscr{Y}_{r, \mu}$, let $\boldsymbol{a}=\left(\mathrm{s}_{1}, \ldots, \mathrm{s}_{p}\right) \in\left(\mathbb{C}^{*}\right)^{r}$ be the $r$-tuple obtained by juxtaposing the segments in $s$. For $1 \leqslant j \leqslant p$ let $\mathfrak{I}_{\mu, j}$ be the left ideal of $\widetilde{\mathscr{H}}_{\mu, j}$ generated by $X_{k}-a_{k}$ for $\mu_{[1, j-1]}+1 \leqslant k \leqslant \mu_{[1, j]}$. Let $\iota_{j}: \mathscr{H}_{\mu, j} \rightarrow \widetilde{\mathscr{H}}_{\mu, j} / \mathfrak{I}_{\mu, j}$ be the natural $\mathscr{H}_{\mu, j}$-module isomorphism defined by sending $h$ to $\bar{h}$. Let

$$
\overline{\mathscr{I}}_{\mu, j}=\iota_{j}\left(\mathscr{I}_{\mu, j}\right)=\mathscr{H}_{\mu, j} \bar{y}_{\mu^{(j)}}=\tilde{\mathscr{H}}_{\mu, j} \bar{y}_{\mu^{(j)}} .
$$

By Lemma 4.3 we have the following corollary.

Corollary 4.4. Maintain the notation above. There is a $\mathrm{U}_{\mathbb{C}}\left(\mathfrak{g l}_{n}\right)$-module isomorphism

$$
\varphi:\left(\Omega_{\mathbb{C}}^{\otimes \mu_{1}} \otimes \widetilde{\mathscr{H}}_{\mu, 1} \bar{\Phi}_{\mu, 1}\right) \otimes \cdots \otimes\left(\Omega_{\mathbb{C}}^{\otimes \mu_{p}} \otimes \widetilde{\mathscr{H}}_{\mu, p} \bar{\Phi}_{\mu, p}\right) \rightarrow \mathrm{F}\left(\overline{\mathscr{I}}_{\mu}\right)
$$


such that $\varphi\left(w_{1} \otimes \bar{h}_{1} \otimes \cdots \otimes w_{p} \otimes \bar{h}_{p}\right)=w_{1} \otimes \cdots \otimes w_{p} \otimes \overline{h_{1} \cdots h_{p}}$ for $w_{j} \in \Omega_{n, \mathbb{C}}^{\otimes \mu_{j}}$ and $h_{j} \in \mathscr{I}_{\mu, j}$ with $1 \leqslant j \leqslant p$.

Proof. Combining Lemmas 3.1, 4.1 with 4.2 yields $\mathrm{F}\left(\bar{\Phi}_{\mu}\right) \cong \Omega_{n, \mathbb{C}}^{\otimes r} \otimes_{\mathscr{H}(r)_{\mathbb{C}}} \bar{\Phi}_{\mu} \cong$

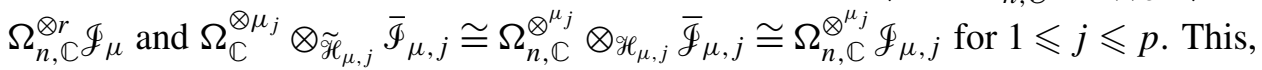
together with Lemma 4.3, implies the assertion.

We now prove that $\varphi$ is in fact a $\mathrm{U}_{\mathbb{C}}\left(\widehat{\mathfrak{g l}}_{n}\right)$-module isomorphism.

Lemma 4.5. The map $\varphi$ is a $\mathrm{U}_{\mathbb{C}}\left(\widehat{\mathfrak{g l}}_{n}\right)$-module homomorphism.

Proof. Let $u \in \mathrm{U}_{\mathbb{C}}\left(\widehat{\mathfrak{g l}}_{n}\right)$ and $w=w_{1} \otimes \bar{h}_{1} \otimes \cdots \otimes w_{p} \otimes \bar{h}_{p} \in\left(\Omega_{\mathbb{C}}^{\otimes \mu_{1}} \otimes \widetilde{\mathscr{H}}_{\mu, 1} \bar{\Phi}_{\mu, 1}\right) \otimes$ $\cdots \otimes\left(\Omega_{\mathbb{C}}^{\otimes \mu_{p}} \otimes \widetilde{\mathscr{H}}_{\mu, p} \bar{\Phi}_{\mu, p}\right)$, where $w_{i} \in \Omega_{n, \mathbb{C}}^{\otimes \mu_{i}}$ and $h_{i} \in \mathscr{I}_{\mu, i}$ for $1 \leqslant i \leqslant p$. Assume $\Delta^{(p-1)}(u)=\sum_{(u)} u_{1} \otimes \cdots \otimes u_{p}, u_{i} w_{i}=\sum_{k_{i}} w_{i, k_{i}} g_{i, k_{i}}$ and $g_{i, k_{i}} h_{i}=\sum_{j_{i}} g_{i, k_{i}, j_{i}} X_{j_{i}}$, where $w_{i, k_{i}} \in \Omega_{n, \mathbb{C}}^{\otimes \mu_{i}}, g_{i, k_{i}} \in \widetilde{\mathscr{H}}_{\mu, i}$, and $g_{i, k_{i}, j_{i}} \in \mathscr{H}_{\mu, i}, X_{j_{i}} \in \widetilde{\mathscr{H}}_{\mu, i}$. Then

$$
g_{i, k_{i}}\left(\iota_{i}\left(h_{i}\right)\right)=g_{i, k_{i}} \overline{h_{i}}=\sum_{j_{i}} a_{j_{i}} \overline{g_{i, k_{i}, j_{i}}} .
$$

Hence,

Since

$$
\begin{aligned}
u w & =\sum_{(u)} u_{1} w_{1} \otimes \bar{h}_{1} \otimes \cdots \otimes u_{p} w_{p} \otimes \bar{h}_{p} \\
& =\sum_{(u)} \sum_{k_{1}, \ldots, k_{p}} w_{1, k_{1}} \otimes g_{1, k_{1}} \bar{h}_{1} \otimes \cdots \otimes w_{p, k_{p}} \otimes g_{p, k_{p}} \bar{h}_{p} \\
& =\sum_{(u)} \sum_{\substack{k_{1}, \ldots, k_{p} \\
j_{1}, \ldots, j_{p}}} a_{j_{1}} \cdots a_{j_{p}} w_{1, k_{1}} \otimes \overline{g_{1, k_{1}, j_{1}}} \otimes \cdots \otimes w_{p, k_{p}} \otimes \overline{g_{p, k_{p}, j_{p}}} .
\end{aligned}
$$

$g_{1, k_{1}} \cdots g_{p, k_{p}} \overline{h_{1} \cdots h_{p}}=\overline{g_{1, k_{1}} h_{1} \cdots g_{p, k_{p}} h_{p}}=\sum_{j_{1}, \ldots, j_{p}} a_{j_{1}} \cdots a_{j_{p}} \overline{g_{1, k_{1}, j_{1}} \cdots g_{p, k_{p}, j_{p}}}$,

we conclude that

$$
\begin{aligned}
\varphi(u w) & =\sum_{(u)} \sum_{\substack{k_{1}, \ldots, k_{p} \\
j_{1}, \ldots, j_{p}}} a_{j_{1}} \cdots a_{j_{p}} w_{1, k_{1}} \otimes \cdots \otimes w_{p, k_{p}} \otimes \overline{g_{1, k_{1}, j_{1}} \cdots g_{p, k_{p}, j_{p}}} \\
& =\sum_{(u)} \sum_{k_{1}, \ldots, k_{p}} w_{1, k_{1}} \otimes \cdots \otimes w_{p, k_{p}} \otimes g_{1, k_{1}} \cdots g_{p, k_{p}} \overline{h_{1} \cdots h_{p}} \\
& =\sum_{(u)} u_{1} w_{1} \otimes \cdots \otimes u_{p} w_{p} \otimes \overline{h_{1} \cdots h_{p}} \\
& =u\left(w_{1} \otimes \cdots \otimes w_{p} \otimes \overline{h_{1} \cdots h_{p}}\right) \\
& =u \varphi(w) .
\end{aligned}
$$


We can now describe $F\left(\bar{\Phi}_{\wp(s)}\right)$ as follows.

Proposition 4.6. Let $s=\left\{\mathrm{s}_{1}, \ldots, \mathrm{s}_{p}\right\} \in \mathscr{S}_{r, \mu}$ with $\mathrm{s}_{i}=\left(a_{i} v^{-\mu_{i}+1}, a_{i} v^{-\mu_{i}+3}, \ldots\right.$, $\left.a_{i} v^{\mu_{i}-1}\right)$. Then $\mathrm{F}\left(\bar{\Phi}_{\mu}\right)=0$ for $\boldsymbol{s} \notin \mathscr{Y}_{r}^{(n)}$ and $\mathrm{F}\left(\bar{\Phi}_{\mu}\right) \cong L\left(\boldsymbol{Q}_{1}\right) \otimes \cdots \otimes L\left(\boldsymbol{Q}_{p}\right)$ for $\boldsymbol{s} \in \mathscr{S}_{r}^{(n)}$, where $\boldsymbol{Q}_{i}=\left(Q_{i, 1}(u), \ldots, Q_{i, n}(u)\right)$ with $Q_{i, n}(u)=\left(1-a_{i} v^{-n+1} u\right)^{\delta_{\mu_{i}, n}}$ and $Q_{i, j}\left(u v^{j-1}\right) / Q_{i, j+1}\left(u v^{j+1}\right)=\left(1-a_{i} u\right)^{\delta_{j, \mu_{i}}}$ for $1 \leqslant i \leqslant p$ and $1 \leqslant j \leqslant n-1$.

Proof. Since $\bar{\Phi}_{\mu_{i}} \cong V_{\mathrm{s}_{i}}$ for $1 \leqslant i \leqslant p$, by Corollary 4.4 and Lemma 4.5 we conclude that $\mathrm{F}\left(\bar{\Phi}_{\mu}\right)=\mathrm{F}_{n, r}\left(\bar{\Phi}_{\mu}\right) \cong \mathrm{F}_{n, \mu_{1}}\left(V_{\mathrm{s}_{1}}\right) \otimes \cdots \otimes \mathrm{F}_{n, \mu_{p}}\left(V_{\mathrm{s}_{p}}\right)$. If $s \notin \mathscr{Y}_{r}^{(n)}$, then there exists $1 \leqslant k \leqslant p$ such that $\left|\mathrm{s}_{k}\right|=\mu_{k}>n$. By Lemma 3.3 we have $\mathrm{F}_{n, \mu_{k}}\left(V_{\mathrm{s}_{k}}\right)=0$ and hence $\mathrm{F}\left(\overline{\mathscr{T}}_{\mu}\right)=0$. If $\boldsymbol{s} \in \mathscr{Y}_{r}^{(n)}$, then by Lemma 3.4 we have $\mathrm{F}_{n, \mu_{i}}\left(V_{\mathrm{s}_{i}}\right) \cong L\left(\boldsymbol{Q}_{i}\right)$ for $1 \leqslant i \leqslant p$. Consequently, $\mathrm{F}\left(\bar{\Phi}_{\mu}\right) \cong L\left(\boldsymbol{Q}_{1}\right) \otimes \cdots \otimes L\left(\boldsymbol{Q}_{p}\right)$.

We now turn to studying $\mathrm{F}\left(V_{s}\right)$ for $s \in \mathscr{S}_{r}^{(n)}$. To compute $\mathrm{F}\left(V_{s}\right)$, we need to generalize [Chari and Pressley 1996, §7.2] to the case of $n \leqslant r$. Recall the notation $\Lambda(n, r)$ defined in (3.1.1). Let $\Lambda^{+}(n, r)=\Lambda(n, r) \cap \Lambda^{+}(r)$. For $\lambda \in \mathbb{N}^{n}$ let $L(\lambda)$ be the irreducible $\mathrm{U}_{\mathbb{C}}\left(\mathfrak{g l}_{n}\right)$-module with highest weight $\lambda$. For $1 \leqslant i \leqslant n$, let $\mathfrak{k}_{i}=\zeta_{r}\left(K_{i}\right)$ and

$$
\left[\begin{array}{c}
\mathfrak{k}_{i} ; 0 \\
t
\end{array}\right]=\prod_{s=1}^{t} \frac{\mathfrak{k}_{i} v^{-s+1}-\mathfrak{k}_{i}^{-1} v^{s-1}}{v^{s}-v^{-s}} .
$$

For $\mu \in \mathbb{N}^{n}$ let $\mathfrak{k}_{\mu}=\left[\begin{array}{c}\mathfrak{k}_{1} ; 0 \\ \mu_{1}\end{array}\right] \cdots\left[\begin{array}{c}\mathfrak{k}_{n} ; 0 \\ \mu_{n}\end{array}\right]$. The following result is the generalization of [Chari and Pressley 1996, §7.2].

Lemma 4.7. Let $\mu \in \Lambda^{+}(r)$. Then $\Omega_{n, \mathbb{C}}^{\otimes r} \otimes_{\mathscr{H}(r)_{\mathbb{C}}} E_{\mu} \neq 0$ if and only if $\mu^{\prime} \in$ $\Lambda(n, r)$, where $\mu^{\prime}$ is the dual partition of $\mu$. Furthermore if $\mu^{\prime} \in \Lambda^{+}(n, r)$, then $\Omega_{n, \mathbb{C}}^{\otimes r} \otimes_{\mathscr{H}(r)_{\mathbb{C}}} E_{\mu} \cong L\left(\mu^{\prime}\right)$.

Proof. We choose $N$ such that $N>\max \{n, r\}$. Let $e=\sum_{\mu \in \Lambda(n, r)} \mathfrak{k}_{\mu} \in \mathscr{S}(N, r)_{\mathbb{C}}$. It is well known that for $\mu \in \Lambda^{+}(N, r), e L(\mu) \neq 0$ if and only if $\mu \in \Lambda(n, r)$ (see [Green 2007, (6.5f)]). Furthermore by [Chari and Pressley 1996, §7.2; Deng, Du and Fu 2012, Lemma 4.3.3] we have $\Omega_{n, \mathbb{C}}^{\otimes r} \otimes_{\mathcal{H}(r)_{\mathbb{C}}} E_{\mu} \cong e\left(\Omega_{N, \mathbb{C}}^{\otimes r} \otimes_{\mathcal{H}(r)_{\mathbb{C}}} E_{\mu}\right) \cong e\left(L\left(\mu^{\prime}\right)\right)$. Thus $\Omega_{n, \mathbb{C}}^{\otimes r} \otimes_{\mathscr{H}(r)_{\mathbb{C}}} E_{\mu} \neq 0$ if and only if $\mu^{\prime} \in \Lambda(n, r)$. If $\mu^{\prime} \in \Lambda^{+}(n, r)$, then $\Omega_{n, \mathbb{C}}^{\otimes r} \otimes_{\mathscr{H}(r)_{\mathbb{C}}} E_{\mu} \cong e\left(L\left(\mu^{\prime}\right)\right) \cong L\left(\mu^{\prime}\right)$.

In the case of $n>r$, the Drinfeld polynomials associated with $\mathrm{F}\left(V_{s}\right)$ were calculated for $s \in \mathscr{S}_{r}^{(n)}$ in [Chari and Pressley 1996, §7.6; Deng, Du and Fu 2012, Theorem 4.4.2]. We are now prepared to use Proposition 4.6 and Lemma 4.7 to generalize these results to the case of $n \leqslant r$ in Theorem 4.9 .

Let $2(n)_{r}=\left\{\boldsymbol{Q} \in \mathscr{2}(n) \mid \sum_{1 \leqslant i \leqslant n} \operatorname{deg} Q_{i}(u)=r\right\}$. For $\boldsymbol{s}=\left\{\mathrm{s}_{1}, \ldots, \mathrm{s}_{p}\right\} \in \mathscr{Y}_{r}^{(n)}$ with

$$
\mathrm{s}_{i}=\left(a_{i} v^{-\mu_{i}+1}, a_{i} v^{-\mu_{i}+3}, \ldots, a_{i} v^{\mu_{i}-1}\right) \in\left(\mathbb{C}^{*}\right)^{\mu_{i}},
$$


define $Q_{s}=\left(Q_{1}(u), \ldots, Q_{n}(u)\right)$ by setting $Q_{n}(u)=\prod_{\substack{1 \leqslant i \leqslant p \\ \mu_{i}=n}}\left(1-a_{i} u v^{-n+1}\right)$ and

$$
Q_{i}(u)=P_{i}\left(u v^{-i+1}\right) P_{i+1}\left(u v^{-i+2}\right) \cdots P_{n-1}\left(u v^{n-2 i}\right) Q_{n}\left(u v^{2(n-i)}\right)
$$

for $1 \leqslant i \leqslant n-1$, where

$$
P_{i}(u)=\prod_{\substack{1 \leqslant j \leqslant p \\ \mu_{j}=i}}\left(1-a_{j} u\right)
$$

Then

$$
\sum_{1 \leqslant i \leqslant n} \operatorname{deg} Q_{i}(u)=n \operatorname{deg} Q_{n}(u)+\sum_{1 \leqslant i \leqslant n-1} i \operatorname{deg} P_{i}(u)=\sum_{1 \leqslant i \leqslant p} \mu_{i}=r .
$$

So $\boldsymbol{Q}_{s} \in 2(n)_{r}$. Consequently, we obtain a map $\partial_{n, r}: \mathscr{Y}_{r}^{(n)} \rightarrow 2(n)_{r}$ defined by sending $\boldsymbol{s}$ to $\boldsymbol{Q}_{\boldsymbol{s}}$.

Lemma 4.8. The map $\partial_{n, r}: \mathscr{Y}_{r}^{(n)} \rightarrow 2(n)_{r}$ is bijective.

Proof. It is clear that $\partial_{n, r}$ is injective. Let $\boldsymbol{Q}=\left(Q_{1}(u), \ldots, Q_{n}(u)\right) \in 2(n)_{r}$ and let $\lambda \in \Lambda(n, r)$, with $\lambda_{i}=\operatorname{deg} Q_{i}(u)$. For $1 \leqslant j \leqslant n-1$ let

$$
P_{j}(u)=\frac{Q_{j}\left(u v^{j-1}\right)}{Q_{j+1}\left(u v^{j+1}\right)}
$$

and $v_{j}=\operatorname{deg} P_{j}(u)=\lambda_{j}-\lambda_{j+1}$. We write, for $1 \leqslant i \leqslant n-1$,

$$
P_{i}(u)=\left(1-a_{v_{1}+\cdots+v_{i-1}+1} u\right)\left(1-a_{v_{1}+\cdots+v_{i-1}+2} u\right) \cdots\left(1-a_{v_{1}+\cdots+v_{i-1}+v_{i}} u\right),
$$

and $Q_{n}(u)=\left(1-b_{1} u\right) \cdots\left(1-b_{\lambda_{n}} u\right)$. Let $p^{\prime}=\sum_{1 \leqslant i \leqslant n-1} v_{i}$ and $p=p^{\prime}+\lambda_{n}$. Let $\boldsymbol{s}=\left\{\mathrm{s}_{1}, \ldots, \mathrm{s}_{p}\right\}$, where

$$
\mathrm{s}_{i}= \begin{cases}\left(a_{i} v^{-\mu_{i}+1}, a_{i} v^{-\mu_{i}+3}, \ldots, a_{i} v^{\mu_{i}-1}\right) & \text { for } 1 \leqslant i \leqslant p^{\prime}, \\ \left(b_{i-p^{\prime}}, b_{i-p^{\prime}} v^{2}, \ldots, b_{i-p^{\prime}} v^{2(n-1)}\right) & \text { for } p^{\prime}+1 \leqslant i \leqslant p,\end{cases}
$$

and $\left(\mu_{1}, \ldots, \mu_{p^{\prime}}\right)=\left(1^{v_{1}}, \ldots,(n-1)^{v_{n-1}}\right)$. Since

$$
\sum_{1 \leqslant i \leqslant p}\left|s_{i}\right|=\sum_{1 \leqslant j \leqslant p^{\prime}} \mu_{j}+n \lambda_{n}=\sum_{1 \leqslant i \leqslant n-1} i v_{i}+n \lambda_{n}=\sum_{1 \leqslant i \leqslant n} \lambda_{i}=r,
$$

we have $s \in \mathscr{Y}_{r}^{(n)}$. It is easy to see that $\partial_{n, r}(s)=\boldsymbol{Q}$. Thus $\partial_{n, r}$ is surjective.

Theorem 4.9. For $s=\left\{\mathrm{s}_{1}, \ldots, \mathrm{s}_{p}\right\} \in \mathscr{S}_{r}^{(n)}$ with $\mathrm{s}_{i}=\left(a_{i} v^{-\mu_{i}+1}, a_{i} v^{-\mu_{i}+3}, \ldots\right.$, $\left.a_{i} v^{\mu_{i}-1}\right)$, we have $\mathrm{F}\left(V_{\boldsymbol{s}}\right) \cong L\left(\boldsymbol{Q}_{s}\right)$, where $\boldsymbol{Q}_{\boldsymbol{s}}=\partial_{n, r}(\boldsymbol{s})$. In particular, we have $\left.\mathrm{F}\left(V_{s}\right)\right|_{\mathrm{U}_{\mathbb{C}}\left(\widehat{\mathfrak{s}}_{n}\right)} \cong \bar{L}(\boldsymbol{P})$, where

$$
P_{i}(u)=\prod_{\substack{1 \leqslant j \leqslant p \\ \mu_{j}=i}}\left(1-a_{j} u\right) \quad \text { for } 1 \leqslant i \leqslant n-1 .
$$


Proof. Let $W=\mathrm{F}\left(\bar{\Phi}_{\mu}\right)$. By Proposition 4.6 we have $W \cong L\left(\boldsymbol{Q}_{1}\right) \otimes \cdots \otimes L\left(\boldsymbol{Q}_{p}\right)$, where $\boldsymbol{Q}_{i}=\left(Q_{i, 1}(u), \ldots, Q_{i, n}(u)\right)$ with $Q_{i, n}(u)=\left(1-a_{i} v^{-n+1} u\right)^{\delta_{i, n}}$ and

$$
P_{i, j}(u):=\frac{Q_{i, j}\left(u v^{j-1}\right)}{Q_{i, j+1}\left(u v^{j+1}\right)}=\left(1-a_{i} u\right)^{\delta_{j, \mu_{i}}}
$$

for $1 \leqslant i \leqslant p$ and $1 \leqslant j \leqslant n-1$. We will identify $W$ with $L\left(\boldsymbol{Q}_{1}\right) \otimes \cdots \otimes L\left(\boldsymbol{Q}_{p}\right)$. Let $w=w_{1} \otimes \cdots \otimes w_{p} \in W$, where $w_{i}$ is the pseudo-highest weight vector in $L\left(\boldsymbol{Q}_{i}\right)$. Then by [Chari and Pressley 1996, §6.3; Frenkel and Mukhin 2002, Lemma 4.1] we conclude that $w$ is the pseudo-highest weight vector in $W$ such that $\mathrm{k}_{i} w=v^{\lambda_{i}} w$ and $2_{i}^{ \pm}(u) w=Q_{i}^{ \pm}(u) w$ for $1 \leqslant i \leqslant n$, where $\lambda_{i}=\operatorname{deg} Q_{i}^{+}(u)$,

$$
\begin{aligned}
Q_{n}^{ \pm}(u) & =\prod_{1 \leqslant i \leqslant p} Q_{i, n}^{ \pm}(u)=\prod_{1 \leqslant i \leqslant p}\left(1-\left(a_{i} u\right)^{ \pm 1} v^{ \pm(-n+1)}\right)^{\delta_{\mu_{i}, n}} \\
& =\prod_{\substack{1 \leqslant i \leqslant p \\
\mu_{i}=n}}\left(1-\left(a_{i} u\right)^{ \pm 1} v^{ \pm(-n+1)}\right)
\end{aligned}
$$

and

$$
\begin{aligned}
P_{j}^{ \pm}(u) & :=\frac{Q_{j}^{ \pm}\left(v^{j-1} u\right)}{Q_{j+1}^{ \pm}\left(v^{j+1} u\right)}=\prod_{1 \leqslant i \leqslant p} P_{i, j}^{ \pm}(u) \\
& =\prod_{1 \leqslant i \leqslant p}\left(1-\left(a_{i} u\right)^{ \pm 1}\right)^{\delta_{j, \mu_{i}}}=\prod_{\substack{1 \leqslant i \leqslant p \\
\mu_{i}=j}}\left(1-\left(a_{i} u\right)^{ \pm 1}\right)
\end{aligned}
$$

for $1 \leqslant j \leqslant n-1$. By definition we have $\boldsymbol{Q}_{s}=\left(Q_{1}^{+}(u), \ldots, Q_{n}^{+}(u)\right)$. Since

$$
\lambda_{j}=\operatorname{deg} Q_{j}^{+}(u)=\lambda_{n}+\sum_{j \leqslant s \leqslant n-1} \operatorname{deg} P_{s}^{+}(u)=\left|\left\{1 \leqslant i \leqslant p \mid \mu_{i} \geqslant j\right\}\right|
$$

for $1 \leqslant j \leqslant n$, we have $\lambda=\left(\lambda_{1}, \ldots, \lambda_{n}\right)=\mu^{\prime}$.

Let $L=\mathrm{F}\left(V_{s}\right)$. Since $V_{s}$ is a semisimple $\mathscr{H}(r)_{\mathbb{C}}$-module, by Lemmas 3.1 and 4.7 we have $[L: L(\lambda)]=\left[L: \Omega_{n, \mathbb{C}}^{\otimes r} \otimes_{\mathscr{H}(r)_{\mathbb{C}}} E_{\mu}\right]=\left[\Omega_{n, \mathbb{C}}^{\otimes r} \otimes_{\mathscr{H}(r)_{\mathbb{C}}} V_{s}: \Omega_{n, \mathbb{C}}^{\otimes r} \otimes_{\mathscr{H}(r)_{\mathbb{C}}} E_{\mu}\right]=$ $\left[V_{s}: E_{\mu}\right]=1$. Thus

$$
\operatorname{dim} L_{\lambda}=1 \text {. }
$$

Since $V_{s}$ is the irreducible subquotient of $\bar{\Phi}_{\mu}$, there is a surjective $\mathrm{U}_{\mathbb{C}}\left(\widehat{\mathfrak{g l}}_{n}\right)$-module homomorphism $f: M \rightarrow L$, where $M$ is a certain submodule of $W$. Since $1=$ $\operatorname{dim} L_{\lambda} \leqslant \operatorname{dim} M_{\lambda} \leqslant \operatorname{dim} W_{\lambda}=1$, we conclude that $\operatorname{dim} M_{\lambda}=\operatorname{dim} W_{\lambda}=1$. Hence $M_{\lambda}=W_{\lambda}=\operatorname{span}\{w\}$ and $L_{\lambda}=\operatorname{span}\{f(w)\}$. By (4.9.1) we have $f(w) \neq 0$. Since $f$ is a $\mathrm{U}_{\mathbb{C}}\left(\widehat{\mathfrak{g l}}_{n}\right)$-module homomorphism, $f(w)$ is the pseudo-highest weight vector in $L$ such that $\mathrm{k}_{i} f(w)=f\left(\mathrm{k}_{i} w\right)=v^{\lambda_{i}} f(w)$ and $2_{i}^{ \pm}(u) f(w)=f\left(2_{i}^{ \pm}(u) w\right)=$ $Q_{i}^{ \pm}(u) f(w)$ for $1 \leqslant i \leqslant n$. This implies that $L$ is the irreducible quotient module of $M\left(\boldsymbol{Q}_{s}\right)$ and hence $L \cong L\left(\boldsymbol{Q}_{s}\right)$. 
Combining Lemmas 3.3, 4.8 with 4.9 yields the following classification theorem of irreducible $\mathscr{S}_{\Delta}(n, r)_{\mathbb{C}}$-modules, which was proved as Theorem 4.6.8 in [Deng, $\mathrm{Du}$ and $\mathrm{Fu}$ 2012] using a different approach.

Corollary 4.10. The set $\left\{L(\boldsymbol{Q}) \mid \boldsymbol{Q} \in 2(n)_{r}\right\}$ is a complete set of nonisomorphic finite-dimensional irreducible $\mathscr{S}_{\Delta}(n, r)_{\mathbb{C}}$-modules.

Finally we will use Theorem 4.9 to generalize [Green 2007, (6.5f)] to the affine case in Theorem 4.11. Assume $N \geqslant n$. Let $e=\sum_{\lambda \in \Lambda(n, r)} \mathfrak{k}_{\lambda} \in \mathscr{S}_{\Delta}(N, r)_{\mathbb{C}}$. Then $e \mathscr{S}_{\Delta}(N, r)_{\mathbb{C}} e \cong \mathscr{Y}_{\Delta}(n, r)_{\mathbb{C}}$. Consequently, the categories $e \mathscr{Y}_{\Delta}(N, r)_{\mathbb{C}} e$-mod and

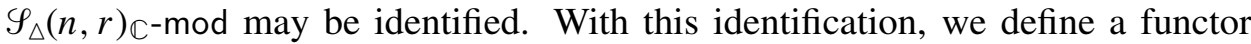

$$
\mathrm{G}=\mathrm{G}_{N, n, r}: \mathscr{Y}_{\Delta}(N, r)_{\mathbb{C}-\bmod } \rightarrow \mathscr{Y}_{\Delta}(n, r)_{\mathbb{C}}-\bmod , \quad V \mapsto e V .
$$

Then by definition we have $\mathrm{G}_{N, n, r} \circ \mathrm{F}_{N, r}=\mathrm{F}_{n, r}$. For $\boldsymbol{Q}=\left(Q_{1}(u), \ldots, Q_{n}(u)\right) \in$ $2(n)_{r}$ let $\widetilde{\boldsymbol{Q}}=\left(Q_{1}(u), \ldots, Q_{n}(u), 1, \ldots, 1\right) \in \mathscr{2}(N)_{r}$. Let $\widetilde{\mathscr{Q}}(n)_{r}=\left\{\widetilde{\boldsymbol{Q}} \mid \boldsymbol{Q} \in \mathscr{2}(n)_{r}\right\}$ $\subseteq 2(N)_{r}$. Clearly, by definition, we have

$$
\partial_{N, r}(s)=\widetilde{\partial_{n, r}(s)} \text { for } s \in \mathscr{S}_{r}^{(n)} \text {. }
$$

Theorem 4.11. Assume $N \geqslant n$. Then $\mathrm{G}(L(\widetilde{\boldsymbol{Q}})) \cong L(\boldsymbol{Q})$ for $\boldsymbol{Q} \in 2(n)_{r}$. In particular we have $\operatorname{dim} L(\widetilde{\boldsymbol{Q}})_{\alpha}=\operatorname{dim} L(\boldsymbol{Q})_{\alpha}$ for $\alpha \in \Lambda(n, r)$. Furthermore, for $\boldsymbol{Q}^{\prime} \in \mathscr{2}(N)_{r}, \mathrm{G}\left(L\left(\boldsymbol{Q}^{\prime}\right)\right) \neq 0$ if and only if $\boldsymbol{Q}^{\prime} \in \widetilde{2}(n)_{r}$.

Proof. If $Q \in 2(n)_{r}$ then by Lemma 4.8 we conclude that there exist $\boldsymbol{s} \in \mathscr{Y}_{r}^{(n)}$ such that $\boldsymbol{Q}=\partial_{n, r}(\boldsymbol{s})$. By Theorem 4.9 and (4.10.2) we have $L(\widetilde{\boldsymbol{Q}}) \cong \mathscr{T}_{\Delta}(N, r) \otimes_{\mathscr{H}_{\Delta}(r)_{\mathbb{C}}} V_{\boldsymbol{s}}$. So by [Deng, Du and Fu 2012, Lemma 4.3.3] and Theorem 4.9 we have

$$
\mathrm{G}(L(\widetilde{\boldsymbol{Q}})) \cong\left(e \mathscr{T}_{\Delta}(N, r)\right) \otimes_{\mathscr{H}_{\Delta}(r)_{\mathbb{C}}} V_{\boldsymbol{s}} \cong \mathscr{T}_{\Delta}(n, r) \otimes_{\mathscr{H}_{\Delta}(r)_{\mathbb{C}}} V_{\boldsymbol{s}} \cong L(\boldsymbol{Q}) .
$$

By [Green 2007, $(6.2 \mathrm{~g})]$, the set $\left\{\mathrm{G}\left(L\left(\boldsymbol{Q}^{\prime}\right)\right) \neq 0 \mid \boldsymbol{Q}^{\prime} \in 2(N)_{r}\right\}$ forms a complete set of non-isomorphic irreducible $\mathscr{S}_{\Delta}(n, r)_{\mathbb{C}}$-modules. This together with Corollary 4.10 implies that $\left\{\mathrm{G}\left(L\left(\boldsymbol{Q}^{\prime}\right)\right) \neq 0 \mid \boldsymbol{Q}^{\prime} \in \mathscr{2}(N)_{r}\right\}=\left\{\mathrm{G}(L(\widetilde{\boldsymbol{Q}})) \mid \boldsymbol{Q} \in \mathscr{2}(n)_{r}\right\}$. Consequently, $\mathrm{G}\left(L\left(\boldsymbol{Q}^{\prime}\right)\right) \neq 0$ if and only if $\boldsymbol{Q}^{\prime} \in \widetilde{\mathscr{2}}(n)_{r}$.

\section{References}

[Beck 1994] J. Beck, "Braid group action and quantum affine algebras", Comm. Math. Phys. 165:3 (1994), 555-568. MR 95i:17011 Zbl 0807.17013

[Chari and Pressley 1991] V. Chari and A. Pressley, "Quantum affine algebras", Comm. Math. Phys. 142:2 (1991), 261-283. MR 93d:17017 Zbl 0739.17004

[Chari and Pressley 1994] V. Chari and A. Pressley, A guide to quantum groups, Cambridge University Press, 1994. MR 95j:17010 Zbl 0839.17009

[Chari and Pressley 1995] V. Chari and A. Pressley, "Quantum affine algebras and their representations", pp. 59-78 in Representations of groups (Banff, AB, 1994), edited by B. N. Allison and G. H. Cliff, CMS Conf. Proc. 16, Amer. Math. Soc., Providence, RI, 1995. MR 96j:17009 Zbl 0855.17009 
[Chari and Pressley 1996] V. Chari and A. Pressley, "Quantum affine algebras and affine Hecke algebras”, Pacific J. Math. 174:2 (1996), 295-326. MR 97i:17011 Zbl 0881.17011

[Chari and Pressley 1997] V. Chari and A. Pressley, "Quantum affine algebras at roots of unity", Represent. Theory 1 (1997), 280-328. MR 98e:17018 Zbl 0891.17013

[Deng, Du and Fu 2012] B. Deng, J. Du, and Q. Fu, A double Hall algebra approach to affine quantum Schur-Weyl theory, London Mathematical Society Lecture Note Series 401, Cambridge University Press, 2012. MR 3113018 Zbl 1269.20045

[Frenkel and Mukhin 2002] E. Frenkel and E. Mukhin, "The Hopf algebra Rep $U_{q} \widehat{\mathfrak{g l}}_{\infty}$ ", Selecta Math. (N.S.) 8:4 (2002), 537-635. MR 2003k:17019 Zbl 1034.17009

[Green 2007] J. A. Green, Polynomial representations of $\mathrm{GL}_{n}$, Lecture Notes in Mathematics 830, Springer, Berlin, 2007. MR 2009b:20084 Zbl 1108.20044

[Jimbo 1986] M. Jimbo, "A $q$-analogue of $U(\mathfrak{g l}(N+1))$, Hecke algebra, and the Yang-Baxter equation”, Lett. Math. Phys. 11:3 (1986), 247-252. MR 87k:17011 Zbl 0602.17005

[Kazhdan and Lusztig 1979] D. Kazhdan and G. Lusztig, "Representations of Coxeter groups and Hecke algebras”, Invent. Math. 53:2 (1979), 165-184. MR 81j:20066 Zbl 0499.20035

[Rogawski 1985] J. D. Rogawski, “On modules over the Hecke algebra of a p-adic group”, Invent. Math. 79:3 (1985), 443-465. MR 86j:22028 Zbl 0579.20037

[Zelevinsky 1980] A. V. Zelevinsky, "Induced representations of reductive p-adic groups, II: On irreducible representations of GL(n)", Ann. Sci. École Norm. Sup. (4) 13:2 (1980), 165-210. MR 83g:22012 Zbl 0441.22014

Received May 31, 2013.

QIANG FU

DEPARTMENT OF MATHEMATICS

TONGJI UNIVERSITY

SHANGHAI, 200092

CHINA

q.fu@tongji.edu.cn

q.fu@hotmail.com 


\title{
PACIFIC JOURNAL OF MATHEMATICS
}

\author{
msp.org/pjm
}

Founded in 1951 by E. F. Beckenbach (1906-1982) and F. Wolf (1904-1989)

\section{EDITORS}

Don Blasius (Managing Editor)

Department of Mathematics

University of California

Los Angeles, CA 90095-1555

blasius@math.ucla.edu

\author{
Paul Balmer \\ Department of Mathematics \\ University of California \\ Los Angeles, CA 90095-1555 \\ balmer@math.ucla.edu \\ Robert Finn \\ Department of Mathematics \\ Stanford University \\ Stanford, CA 94305-2125 \\ finn@math.stanford.edu \\ Sorin Popa \\ Department of Mathematics \\ University of California \\ Los Angeles, CA 90095-1555 \\ popa@math.ucla.edu
}

\author{
Vyjayanthi Chari \\ Department of Mathematics \\ University of California \\ Riverside, CA 92521-0135 \\ chari@math.ucr.edu \\ Kefeng Liu \\ Department of Mathematics \\ University of California \\ Los Angeles, CA 90095-1555 \\ liu@math.ucla.edu \\ Jie Qing \\ Department of Mathematics \\ University of California \\ Santa Cruz, CA 95064 \\ qing@ cats.ucsc.edu
}

\section{PRODUCTION}

Silvio Levy, Scientific Editor, production@msp.org

\section{SUPPORTING INSTITUTIONS}

ACADEMIA SINICA, TAIPEI

CALIFORNIA INST. OF TECHNOLOGY

INST. DE MATEMÁTICA PURA E APLICADA

KEIO UNIVERSITY

MATH. SCIENCES RESEARCH INSTITUTE

NEW MEXICO STATE UNIV.

OREGON STATE UNIV.

\author{
STANFORD UNIVERSITY \\ UNIV. OF BRITISH COLUMBIA \\ UNIV. OF CALIFORNIA, BERKELEY \\ UNIV. OF CALIFORNIA, DAVIS \\ UNIV. OF CALIFORNIA, LOS ANGELES \\ UNIV. OF CALIFORNIA, RIVERSIDE \\ UNIV. OF CALIFORNIA, SAN DIEGO \\ UNIV. OF CALIF., SANTA BARBARA
}

\author{
Daryl Cooper \\ Department of Mathematics \\ University of California \\ Santa Barbara, CA 93106-3080 \\ cooper@math.ucsb.edu \\ Jiang-Hua Lu \\ Department of Mathematics \\ The University of Hong Kong \\ Pokfulam Rd., Hong Kong \\ jhlu@maths.hku.hk \\ Paul Yang \\ Department of Mathematics \\ Princeton University \\ Princeton NJ 08544-1000 \\ yang@math.princeton.edu
}

These supporting institutions contribute to the cost of publication of this Journal, but they are not owners or publishers and have no responsibility for its contents or policies.

See inside back cover or msp.org/pjm for submission instructions.

The subscription price for 2014 is US $\$ 410 /$ year for the electronic version, and \$535/year for print and electronic.

Subscriptions, requests for back issues and changes of subscribers address should be sent to Pacific Journal of Mathematics, P.O. Box 4163, Berkeley, CA 94704-0163, U.S.A. The Pacific Journal of Mathematics is indexed by Mathematical Reviews, Zentralblatt MATH, PASCAL CNRS Index, Referativnyi Zhurnal, Current Mathematical Publications and Web of Knowledge (Science Citation Index).

The Pacific Journal of Mathematics (ISSN 0030-8730) at the University of California, c/o Department of Mathematics, 798 Evans Hall \#3840, Berkeley, CA 94720-3840, is published twelve times a year. Periodical rate postage paid at Berkeley, CA 94704, and additional mailing offices. POSTMASTER: send address changes to Pacific Journal of Mathematics, P.O. Box 4163, Berkeley, CA 94704-0163.

PJM peer review and production are managed by EditFLOW ${ }^{\circledR}$ from Mathematical Sciences Publishers.

\section{PUBLISHED BY}

\section{mathematical sciences publishers \\ nonprofit scientific publishing}

http://msp.org/

(C) 2014 Mathematical Sciences Publishers 


\section{PACIFIC JOURNAL OF MATHEMATICS}

Volume $270 \quad$ No. $2 \quad$ August 2014

Disjointification inequalities in symmetric quasi-Banach spaces and 257 their applications

SERgey Astashinin, Fedor A. SukocheV and DMitriy

ZANIN

Hamiltonian evolutions of twisted polygons in parabolic manifolds:

287

The Lagrangian Grassmannian

GLORIA MARÍ BEFFA

On Schwarz-Christoffel mappings

MARTIN CHUAQUi and CHRISTIAN POMMERENKE

Vanishing viscosity in the plane for nondecaying velocity and vorticity, II

ELAINE COZZI

Affine quantum Schur algebras and affine Hecke algebras

QIANG FU

On the classification of Killing submersions and their isometries

JosÉ M. MANZANO

Locally Lipschitz contractibility of Alexandrov spaces and its applications

Ayato Mitsuishi and TAKaO Yamaguchi

Sequences of open Riemannian manifolds with boundary

RAQUEL PERALES and CHRISTINA SORMANI

Invariant differential operators on a class of multiplicity-free spaces 